\title{
A family of level-dependent biorthogonal wavelet filters for image compression
}

\author{
Vittoria Bruni ${ }^{\mathrm{a}}$, Mariantonia Cotronei $^{\mathrm{b}}$, Francesca Pitolli ${ }^{\mathrm{a}}$ \\ ${ }^{a}$ Department SBAI, Università di Roma "La Sapienza", Italy \\ ${ }^{b}$ DIIES, Università Mediterranea di Reggio Calabria, Italy
}

\begin{abstract}
In this work we explore the construction and the applications of a special family of level-dependent biorthogonal filters, i.e. filters whose taps depend on the scaling level. Such a family is generated from a class of functions all related through level-dependent (or nonstationary) refinement equations, which contains cardinal polynomial B-splines as a particular case. The greater flexibility offered by the nonstationarity of these filters allows to achieve better results in some image processing problems, such as image compression, when compared to classical biorthogonal B-spline filters.
\end{abstract}

Keywords: Level-dependent multiresolution analysis, Biorthogonal wavelets, Nonstationary subdivision, Image compression

2010 MSC: 65T60, 65D15, 41A05

\section{Introduction}

Wavelet analysis is nowadays a well-established tool for signal and image processing problems.

Recently, techniques from the theory of nonstationary subdivision schemes have been used for constructing wavelet bases generating level-dependent (or nonstationary) multiresolution analyses $[3,10,13]$. In such a framework, the decomposition and reconstruction steps of the discrete wavelet transform are based on low-pass and high pass filter sequences which change level by level.

In this paper, we present a special construction of biorthogonal families of wavelet systems constructed from the class of nonstationary refinable functions introduced in $[10,11]$.

The level-dependent nature of such filterbanks provides more flexibility when applied to practical contexts of image processing, since the parameter controlling the nonstationary filters can be properly tuned for adapting the filters to the specific task.

Email addresses: vittoria.bruni@sbai.uniroma1.it (Vittoria Bruni), mariantonia.cotronei@unirc.it (Mariantonia Cotronei), francesca.pitolli@sbai.uniroma1.it (Francesca Pitolli) 
In fact, the preliminary results of our numerical tests confirm that the new filters outperform the B-spline filters, which can be seen as special instances obtained for limiting values of the parameters involved in the masks. In particular, they show higher correlation with human perception when used for image processing purposes.

The paper is organized as follows. In Section 2 we fix the notation and recall some basic facts concerning biorthogonal filters. In Section 3 we introduce our starting low-pass filters and illustrate a procedure for completing them to a biorthogonal system. In Section 4 we provide some applications in image compression which underline the effectiveness of such level-dependent filterbanks in image compaction.

\section{Preliminaries}

Basic notions on nonstationary subdivision schemes and refinable functions can be found, for instance, in [7]. In this section we just recall some definitions and properties of level-dependent multiresolution analyses taken from [11] (see also [13]).

Let us denote by $\ell(\mathbb{Z})$ the space of all sequences. A nonstationary subdivision scheme $S\left(a^{(m)}: m \geq 0\right)$ consists of the successive applications of several subdivision operators, each depending on the mask $a^{(m)} \in \ell(\mathbb{Z})$, which, starting from an initial sequence $c^{(0)} \in \ell(\mathbb{Z})$, generates a sequence of sequences $\left(c^{(m)}: m \geq 0\right)$ as follows:

$$
c_{j}^{(m+1)}=\sum_{k \in \mathbb{Z}} a_{j-2 k}^{(m)} c_{k}^{(m)}, \quad j \in \mathbb{Z}, \quad m \geq 0 .
$$

The scheme (1) is said to be convergent if, for any initial sequence, there exists a uniformly continuous function $f$ such that the sequences $\left(c^{(m)}: m \geq 0\right)$ satisfy

$$
\sup _{j \in \mathbb{Z}}\left|c_{j}^{(m)}-f\left(2^{-m} j\right)\right| \rightarrow 0, \text { as } m \rightarrow \infty .
$$

In such a case, the special choice of the delta sequence $\delta=\left(\delta_{0 j}: j \in \mathbb{Z}\right)$ as initial data produces, in the limit, the so-called basic limit function $\varphi$ of the scheme. Furthermore, all the schemes $S\left(a^{(m)}: m \geq n\right)$ for $n \geq 0$ are convergent, each

with basic limit function $\varphi^{(n)}$, where $\varphi^{(0)}$ coincides with $\varphi$. All these scaling functions are related by the refinement equations

$$
\varphi^{(n)}=\sum_{k \in \mathbb{Z}} a_{k}^{(n)} \varphi^{(n+1)}(2 \cdot-k), \quad n \geq 0 .
$$

This refinement property allows to consider a level-dependent (or nonstationary) multiresolution analysis (MRA) in $L^{2}(\mathbb{R})$, where each space $V_{n}$ is spanned by the translates of the function $\varphi^{(n)}\left(2^{n}\right.$.), i.e.

$$
V_{n}=\overline{\operatorname{span}\left\{\varphi^{(n)}\left(2^{n} \cdot-k\right): k \in \mathbb{Z}\right\}}, \quad n \geq 0 .
$$


Let us now introduce the functions $\widetilde{\varphi}^{(n)}, n \geq 0$, duals to $\varphi^{(n)}$ in the following sense:

$$
\left\langle\varphi^{(n)}, \widetilde{\varphi}^{(n)}(\cdot-k)\right\rangle=\delta_{0 k}, \quad k \in \mathbb{Z}
$$

and satisfying the refinement equations

$$
\widetilde{\varphi}^{(n)}=\sum_{k \in \mathbb{Z}} \tilde{a}_{k}^{(n)} \widetilde{\varphi}^{(n+1)}(2 \cdot-k), \quad n \geq 0,
$$

for some mask sequences $\left(\tilde{a}^{(n)}: n \geq 0\right)$. The relation between such masks and the masks in (3) is expressed by the biorthogonality relation

$$
\sum_{k \in \mathbb{Z}} a_{k}^{(n)} \widetilde{a}_{k-2 j}^{(n)}=2 \delta_{0 j}, \quad j \in \mathbb{Z}
$$

which can also be written as:

$$
A^{(n)}(z) \widetilde{A}^{(n)}\left(z^{-1}\right)+A^{(n)}(-z) \widetilde{A}^{(n)}\left(-z^{-1}\right)=4, \quad|z|=1,
$$

where

$$
A^{(n)}(z)=\sum_{k \in \mathbb{Z}} a_{k}^{(n)} z^{k}, \quad z \in \mathbb{C} \backslash\{0\},
$$

denotes the so-called symbol of the mask $a^{(n)}$.

If we denote with $\widetilde{V}_{n}, n \geq 0$, the spaces generated by the shifts of $\widetilde{\varphi}^{(n)}\left(2^{n} \cdot\right)$, then, the sequence $\left\{\widetilde{V}_{n}\right\}_{n \geq 0}$ is a biorthogonal MRA associated to $\left\{V_{n}\right\}_{n \geq 0}$. The wavelet spaces are defined as the complementary spaces:

$$
W_{n}=V_{n+1}-V_{n}, \quad \widetilde{W}_{n}=\widetilde{V}_{n+1}-\widetilde{V}_{n},
$$

satisfying the orthogonality conditions: $\widetilde{W}_{n} \perp V_{n}, W_{n} \perp \widetilde{V}_{n}$. They are generated by the shifts of the $2^{n}$-dilates of the wavelet functions $\psi^{(n)}, \widetilde{\psi}^{(n)}$, respectively, that obey the two-scale relations

$$
\psi^{(n)}=\sum_{k \in \mathbb{Z}} b_{k}^{(n)} \varphi^{(n+1)}(2 \cdot-k), \quad \widetilde{\psi}^{(n)}=\sum_{k \in \mathbb{Z}} \tilde{b}_{k}^{(n)} \widetilde{\varphi}^{(n+1)}(2 \cdot-k), \quad n \geq 0,
$$

where the wavelet mask sequences $\left(b^{(n)}: n \geq 0\right),\left(\tilde{b}^{(n)}: n \geq 0\right)$, have to satisfy:

$$
\sum_{k \in \mathbb{Z}} b_{k}^{(n)} \widetilde{b}_{k-2 j}^{(n)}=2 \delta_{0 j}, \quad \sum_{k \in \mathbb{Z}} a_{k}^{(n)} \widetilde{b}_{k-2 j}^{(n)}=\sum_{k \in \mathbb{Z}} b_{k}^{(n)} \widetilde{a}_{k-2 j}^{(n)}=0,
$$

or equivalently, in terms of symbols,

$$
\begin{aligned}
& B^{(n)}(z) \widetilde{B}^{(n)}\left(z^{-1}\right)+B^{(n)}(-z) \widetilde{B}^{(n)}\left(-z^{-1}\right)=4, \\
& A^{(n)}(z) \widetilde{B}^{(n)}\left(z^{-1}\right)+A^{(n)}(-z) \widetilde{B}^{(n)}\left(-z^{-1}\right)=0, \\
& B^{(n)}(z) \widetilde{A}^{(n)}\left(z^{-1}\right)+B^{(n)}(-z) \widetilde{A}^{(n)}\left(-z^{-1}\right)=0,
\end{aligned}
$$

for $|z|=1$. As usual, the wavelet symbols can be set as:

$$
\widetilde{B}^{(n)}(z)=z A^{(n)}\left(-z^{-1}\right), \quad B^{(n)}(z)=z \widetilde{A}^{(n)}\left(-z^{-1}\right),
$$

so that the last two conditions in (9) are automatically satisfied. 


\section{Level-dependent biorthogonal wavelet systems}

In this section we construct biorthogonal wavelet systems associated to the class of nonstationary subdivision schemes introduced in [5] (see also [9, 11]). Their masks depend not only on the level, but also on an additional parameter which, as we will see, offers extra degrees of freedom and, thus, a great flexibility in practical implementations.

The starting point is the family of stationary subdivision schemes introduced in [8]. The corresponding masks are bell-shaped so that the associated refinable functions are bell-shaped too. The mask coefficients depend on one or more shape parameters that allow to control the shape of the limit function.

In particular, here we consider the subset of masks in the family depending on one parameter. They are associated with the symbols:

$$
S_{N}(z ; \alpha)=\frac{1}{2^{\alpha}} \frac{(1+z)^{N}}{z^{(N-\varepsilon) / 2+1}}\left(z^{2}+2\left(2^{\alpha-N}-1\right) z+1\right), \quad z \in \mathbb{C} \backslash\{0\},
$$

where $N \in \mathbb{N}$ with $N \geq 1, \varepsilon=0$ for $N$ even, $\varepsilon=1$ for $N$ odd. Here, $\alpha \geq 2$ is a real parameter such that $\alpha-N \geq 1$ : when $\alpha$ varies from $N+1$ to $\infty, S_{N}(z ; \alpha)$ varies continuously between the symbol of the B-spline of order $N+2$ and the symbol of the B-spline of order $N$.

To construct subdivision schemes of nonstationary type, we set the free parameter $\alpha$ to have the special form $\alpha=N+1+n^{-\mu}$, where $\mu \in \mathbb{R}$ with $\mu>1$, so that we obtain the following families of symbols which now depend on the refinement level $n$ and on the parameter $\mu$ :

$$
A_{N}(z ; n, \mu)=\frac{1}{2^{N+1+n^{-\mu}}} \frac{(1+z)^{N}}{z^{(N-\varepsilon) / 2+1}}\left(z^{2}+2\left(2^{n^{-\mu}+1}-1\right) z+1\right), z \in \mathbb{C} \backslash\{0\} .
$$

For the sake of notation let us set $\beta=\beta(n, \mu):=2^{n^{-\mu}}$. The symbols (12) satisfy the symmetry condition

$$
A_{N}\left(\frac{1}{z} ; \beta\right)=\frac{1}{z^{\varepsilon}} A_{N}(z ; \beta)
$$

thus giving rise to linear phase filters. The presence of the factor $(1+z)^{N}$ guarantees the polynomial reproduction property of the mask up to the degree $N-1$. Furthermore, since $\lim _{n \rightarrow 0} \beta(n, \mu)=\infty, \lim _{n \rightarrow \infty} \beta(n, \mu)=1$, in the limit cases, the symbols become

$$
\begin{aligned}
A_{N}(z ; \infty) & =\frac{2}{z^{(N-\varepsilon) / 2}}\left(\frac{1+z}{2}\right)^{N}=\frac{1}{z^{(N-\varepsilon) / 2}} B_{N}(z) \\
A_{N}(z ; 1) & =\frac{2}{z^{(N-\varepsilon) / 2+1}}\left(\frac{1+z}{2}\right)^{N+2}=\frac{1}{z^{(N-\varepsilon) / 2+1}} B_{N+2}(z)
\end{aligned}
$$

where we have denoted with $B_{N}(z)$ the B-spline symbol of order $N$. 
For any $N$, the scheme associated to the sequence of symbols $\left\{A_{N}(z ; n, \mu), n \geq\right.$ $0\}$ converges to a set of nonstationary basic limit functions satisfying a set of nonstationary refinement equations of type (3). The properties of these functions have recently been illustrated in $[9,11]$.

Due to the refinability properties of the limit functions, these schemes are associated to level-dependent MRAs. To construct families of dual symbols, we follow the approach proposed in [4] extending it to our more general situation.

Let us start by expressing the symbols (12) in terms of trigonometric polynomials, with the substitution $z=e^{-i \omega}$. One obtains

$$
\begin{aligned}
A_{N}(\omega ; \beta) & =\frac{1}{2^{N+1} \beta e^{i \frac{\omega}{2} \varepsilon}}\left(e^{i \frac{\omega}{2}}+e^{-i \frac{\omega}{2}}\right)^{N}\left(e^{-i \omega}+2(2 \beta-1)+e^{i \omega}\right) \\
& =\frac{1}{2^{N+1} \beta e^{i \frac{\omega}{2} \varepsilon}}\left(2 \cos \frac{\omega}{2}\right)^{N} 2(\cos \omega+2 \beta-1) \\
& =e^{-i \frac{\omega}{2} \varepsilon} \cos ^{N}\left(\frac{\omega}{2}\right) L\left(\sin ^{2} \frac{\omega}{2} ; \beta\right)
\end{aligned}
$$

where

$$
L(y ; \beta)=\frac{2 \beta-2 y}{\beta} .
$$

We require the dual filters to have polynomial reproduction up to some degree $\tilde{N}-1$. This implies that the dual symbols have the form

$$
\widetilde{A}_{N}^{\widetilde{N}}(\omega ; \beta)=e^{-i \frac{\omega}{2} \varepsilon} \cos ^{\widetilde{N}}\left(\frac{\omega}{2}\right) \widetilde{L}\left(\sin ^{2} \frac{\omega}{2} ; \beta\right),
$$

where the polynomial $\widetilde{L}(y ; \beta)$ has to be found.

To this aim, we give an equivalent formulation of the biorthogonality relation (7) on the unit circle in terms of $A_{N}(\omega ; \beta)$ and $\widetilde{A}_{N}^{\widetilde{N}}(\omega ; \beta)$ :

$$
\begin{aligned}
\cos ^{N+\tilde{N}}\left(\frac{\omega}{2}\right) \frac{2 \beta-2 \sin ^{2} \frac{\omega}{2}}{\beta} \widetilde{L}\left(\sin ^{2}\left(-\frac{\omega}{2}\right) ; \beta\right) \\
\quad+\cos ^{N+\tilde{N}}\left(\frac{\omega+\pi}{2}\right) \frac{2 \beta-2 \sin ^{2} \frac{\omega+\pi}{2}}{\beta} \widetilde{L}\left(\sin ^{2}\left(-\frac{\omega+\pi}{2}\right) ; \beta\right)=4,
\end{aligned}
$$

that is

$$
\begin{aligned}
\cos ^{N+\tilde{N}} & \left(\frac{\omega}{2}\right) \frac{\beta-\sin ^{2} \frac{\omega}{2}}{2 \beta} \widetilde{L}\left(\sin ^{2}\left(\frac{\omega}{2}\right) ; \beta\right) \\
& +(-1)^{N+\tilde{N}} \sin ^{N+\tilde{N}}\left(\frac{\omega}{2}\right) \frac{\beta-\cos ^{2} \frac{\omega}{2}}{2 \beta} \widetilde{L}\left(\cos ^{2}\left(\frac{\omega}{2}\right) ; \beta\right)=1 .
\end{aligned}
$$

By setting $y=\sin ^{2}(\omega / 2)$, the following Lemma is then proved.

Lemma 1. Assume that $N+\tilde{N}$ is even. The biorthogonality relation (7) holds true if and only if the polynomial $\widetilde{L}(y ; \beta)$ satisfies the Bézout identity

$$
(1-y)^{q} \frac{\beta-y}{2 \beta} \widetilde{L}(y ; \beta)+y^{q} \frac{\beta-1+y}{2 \beta} \widetilde{L}(1-y ; \beta)=1,
$$


where

$$
q=\frac{N+\tilde{N}}{2} .
$$

The existence of such polynomial of (minimal) degree $q$ is guaranteed by the Bézout theorem and can be found with the Euclidean polynomial division algorithm. Using similar arguments as in [4] and [6], we propose a simpler and explicit construction.

Proposition 2. The polynomial $\widetilde{L}(y ; \beta)$ is given by

$$
\widetilde{L}(y ; \beta)=\sum_{k=0}^{q} \widetilde{l}_{k}(\beta) y^{k},
$$

where

$$
\widetilde{l}_{k}(\beta)= \begin{cases}\frac{2}{\beta^{k}} \sum_{j=0}^{k}\left(\begin{array}{c}
q+j-1 \\
j
\end{array}\right) \beta^{j}, & k=0, \ldots, q-1, \\
\frac{2}{2 \beta-1} \widetilde{l}_{q-1}(\beta), & k=q .\end{cases}
$$

Proof. Let us rewrite (13) as

$$
\begin{aligned}
\widetilde{L}(y ; \beta) & =(1-y)^{-q} \frac{2 \beta}{\beta-y} \\
& -(1-y)^{-q} \frac{2 \beta}{\beta-y} y^{q} \frac{\beta-1+y}{2 \beta} \widetilde{L}(1-y ; \beta)
\end{aligned}
$$

and consider a Taylor expansion of the right-hand side with respect to $y$. To compute the first $q$ terms of the polynomial:

$$
\widetilde{L}(y ; \beta)=\sum_{k=0}^{q} \widetilde{l}_{k} y^{k}
$$

where $\widetilde{l}_{k}=\widetilde{l}_{k}(\beta)$, we simply have to consider the first $q$ terms of the following expansion:

$$
(1-y)^{-q} \frac{2 \beta}{\beta-y}=2 \sum_{k=0}^{q-1}\left(\sum_{j=0}^{k}\left(\begin{array}{c}
q+j-1 \\
j
\end{array}\right)\left(\frac{1}{\beta}\right)^{k-j}\right) y^{k}+y^{q} R(y),
$$

which gives explicit expressions for the coefficients:

$$
\widetilde{l}_{k}=\frac{2}{\beta^{k}} \sum_{j=0}^{k}\left(\begin{array}{c}
q+j-1 \\
j
\end{array}\right) \beta^{j}, \quad k=0, \ldots, q-1 .
$$

It remains to determine the $q$-th coefficient. To compute it, we make use of (13), which we rewrite more explicitly as

$$
\frac{\beta-y}{2 \beta} \sum_{j=0}^{q}\left(\begin{array}{l}
q \\
j
\end{array}\right)(-1)^{j} y^{j} \sum_{k=0}^{q} \widetilde{l}_{k} y^{k}+\frac{\beta-1+y}{2 \beta} y^{q} \sum_{k=0}^{q} \widetilde{l}_{k} \sum_{j=0}^{k}\left(\begin{array}{c}
k \\
j
\end{array}\right)(-1)^{j} y^{j}=1
$$




\begin{tabular}{c|ccccc}
$q$ & $\tilde{l}_{0}$ & $\tilde{l}_{1}$ & $\tilde{l}_{2}$ & $\tilde{l}_{3}$ & $\tilde{l}_{4}$ \\
\hline 1 & 2 & $\frac{4}{2 \beta-1}$ & & & \\
2 & 2 & $\frac{2(1+2 \beta)}{\beta}$ & $\frac{4(1+2 \beta)}{\beta(2 \beta-1)}$ & & \\
3 & 2 & $\frac{2(1+3 \beta)}{\beta}$ & $\frac{2\left(1+3 \beta+6 \beta^{2}\right)}{\beta^{2}}$ & $\frac{4\left(1+3 \beta+6 \beta^{2}\right)}{\beta^{2}(2 \beta-1)}$ & \\
4 & 2 & $\frac{2(1+4 \beta)}{\beta}$ & $\frac{2\left(1+4 \beta+10 \beta^{2}\right)}{\beta^{2}}$ & $\frac{2\left(1+4 \beta+10 \beta^{2}+20 \beta^{3}\right)}{\beta^{3}}$ & $\frac{4\left(1+4 \beta+10 \beta^{2}+20 \beta^{3}\right)}{\beta^{3}(2 \beta-1)}$
\end{tabular}

Table 1: Coefficients of the polynomial $\widetilde{L}(y)$ (cf. (14)) for $q=1, \ldots, 4$.

\begin{tabular}{c|ccc}
$k$ & $\tilde{N}=1$ & $\tilde{N}=3$ & $\tilde{N}=5$ \\
\hline 1,0 & $-1+4 \beta$ & $-18 \beta+64 \beta^{2}-1$ & $-22 \beta+1024 \beta^{3}-300 \beta^{2}-2$ \\
$2,-1$ & -1 & $-1-14 \beta+8 \beta^{2}$ & $176 \beta^{3}-212 \beta^{2}-14 \beta-2$ \\
$3,-2$ & & $-(1+2 \beta)(-1+4 \beta)$ & $-176 \beta^{3}-38 \beta^{2}+17 \beta+3$ \\
$4,-3$ & & $1+2 \beta$ & $-24 \beta^{3}+38 \beta^{2}+21 \beta+3$ \\
$5,-4$ & & & $(-1+4 \beta)\left(6 \beta^{2}+3 \beta+1\right)$ \\
$6,-5$ & & & $-6 \beta^{2}-3 \beta-1$ \\
\hline Denomin. & $2(2 \beta-1)$ & $2^{5} \beta(2 \beta-1)$ & $2^{9} \beta^{2}(2 \beta-1)$
\end{tabular}

Table 2: Dual filters $\left\{\tilde{a}_{k}^{\beta}\right\}$ of the nonstationary filter (12) with $N=1$.

The coefficient of the monomial $y^{2 q}$ in the left-hand side of the previous identity is $\frac{(-1)^{q}(2 \beta-1) \widetilde{l}_{q}}{2 \beta}+\frac{2(-1)^{q-1} \widetilde{l}_{q-1}}{2 \beta}$. After equating it to zero, we get

$$
\widetilde{l}_{q}=\frac{2}{2 \beta-1} \widetilde{l}_{q-1}
$$

A reconversion in terms of the symbol produces:

$$
\widetilde{A_{N}} \widetilde{N}(z ; \beta)=\frac{1}{z^{(\widetilde{N}-\varepsilon) / 2}}\left(\frac{1+z}{2}\right)^{\widetilde{N}} \sum_{n=0}^{q} \frac{\widetilde{l}_{n}}{2^{2 n}}\left(2-\frac{1}{z}-z\right)^{n}
$$

with $\widetilde{l}_{n}, n=0, \ldots, q$, computed as in (14). We observe that the equation above is still valid when $\mu<1$ and, in particular, for negative values of $\mu$. In this case the limit values of $\beta$ are reversed, i.e. $\lim _{n \rightarrow 0} \beta(n, \mu)=1, \lim _{n \rightarrow \infty} \beta(n, \mu)=\infty$, and so are the limit symbols.

Explicit values for the coefficients of the polynomial $\widetilde{L}(y)$ and of the dual filters $\widetilde{A}_{\widetilde{N}}(z ; \beta)$ are given in Tables 1-4. Figures 1 and 2 illustrate the behaviour of some primal and dual filters of the class, respectively in the case of a varying $n$ and in the case of a varying $\beta$.

We finish the section by mentioning that all such dual filters give rise to scaling and wavelet functions that are only formally biorthogonal, in fact it is not 


\begin{tabular}{c|ccc}
$k$ & $\tilde{N}=2$ & $\tilde{N}=4$ & $\tilde{N}=6$ \\
\hline 0 & $8(6 \beta-1) \beta$ & $8\left(90 \beta^{2}-19 \beta-1\right) \beta$ & $16 \beta\left(-1+700 \beta^{3}-162 \beta^{2}-12 \beta\right)$ \\
$1,-1$ & $16 \beta^{2}-10 \beta-1$ & $-14 \beta-2-148 \beta^{2}+304 \beta^{3}$ & $-5-2308 \beta^{3}-210 \beta^{2}+5184 \beta^{4}-36 \beta$ \\
$2,-2$ & $-4(2 \beta+1) \beta$ & $-64 \beta^{2}(2 \beta+1)$ & $-4\left(492 \beta^{3}+246 \beta^{2}-4 \beta-1\right) \beta$ \\
$3,-3$ & $2 \beta+1$ & $26 \beta^{2}-48 \beta^{3}+17 \beta+3$ & $9+60 \beta-1248 \beta^{4}+356 \beta^{3}+266 \beta^{2}$ \\
$4,-4$ & & $4\left(6 \beta^{2}+3 \beta+1\right) \beta$ & $8\left(68 \beta^{3}+34 \beta^{2}+12 \beta+1\right) \beta$ \\
$5,-5$ & & $-1-6 \beta^{2}-3 \beta$ & $160 \beta^{4}-116 \beta^{3}-66 \beta^{2}-28 \beta-5$ \\
$6,-6$ & & & $-4 \beta\left(10 \beta^{2}+4 \beta+1+20 \beta^{3}\right)$ \\
$7,-7$ & & & $10 \beta^{2}+4 \beta+1+20 \beta^{3}$ \\
\hline Denomin. & $2^{4} \beta(2 \beta-1)$ & $2^{8} \beta^{2}(2 \beta-1)$ & $2^{12} \beta^{3}(2 \beta-1)$
\end{tabular}

Table 3: Dual filters $\left\{\tilde{a}_{k}^{\beta}\right\}$ of the nonstationary filter (12) with $N=2$.

\begin{tabular}{c|ccc}
$k$ & $\tilde{N}=1$ & $\tilde{N}=3$ & $\tilde{N}=5$ \\
\hline 1,0 & $4 \beta(6 \beta-1)$ & $4 \beta\left(90 \beta^{2}-19 \beta-1\right)$ & $8 \beta\left(700 \beta^{3}-162 \beta^{2}-12 \beta-1\right)$ \\
$2,-1$ & $-(2 \beta+1)(4 \beta+1)$ & $-56 \beta^{3}-72 \beta^{2}-10 \beta-2$ & $-1012 \beta^{3}-28 \beta-416 \beta^{4}-114 \beta^{2}-5$ \\
$3,-2$ & $2 \beta+1$ & $-2(2 \beta-1)\left(18 \beta^{2}+7 \beta+1\right)$ & $-1552 \beta^{4}+28 \beta^{3}+130 \beta^{2}+32 \beta+5$ \\
$4,-3$ & & $(4 \beta+1)\left(1+6 \beta^{2}+3 \beta\right)$ & $136 \beta^{2}+28 \beta+304 \beta^{4}+328 \beta^{3}+4$ \\
$5,-4$ & & $-1-6 \beta^{2}-3 \beta$ & $240 \beta^{4}-56 \beta^{3}-40 \beta^{2}-20 \beta-4$ \\
$6,-5$ & & $-(4 \beta+1)\left(4 \beta+1+20 \beta^{3}+10 \beta^{2}\right)$ \\
$7,-6$ & & & $4 \beta+1+20 \beta^{3}+10 \beta^{2}$ \\
\hline Denomin. & $2^{3} \beta(2 \beta-1)$ & $2^{7} \beta^{2}(2 \beta-1)$ & $2^{11} \beta^{3}(2 \beta-1)$
\end{tabular}

Table 4: Dual filters $\left\{\tilde{a}_{k}^{\beta}\right\}$ of the nonstationary filter (12) with $N=3$.

even guaranteed that they belong to $L^{2}(\mathbb{R})$. To guarantee the existence of scaling and wavelet functions further conditions have to be verified. Nevertheless, our aim is to apply the filters themselves in an image processing framework, where perfect reconstruction properties at each level and filters phase linearity play a crucial role rather than the existence and the properties of the associated biorthogonal functions.

\section{Level-dependent biorthogonal filterbanks and application to image compression}

Let $\left(a^{(n, \mu)}, b^{(n, \mu)}, \tilde{a}^{(n, \mu)}, \tilde{b}^{(n, \mu)}: n \geq 0, \mu \in \mathbb{R}\right)$ be the biorthogonal wavelet filterbank system obtained as described in the previous section, namely the scal$\operatorname{ing}$ sequences $a^{(n, \mu)}, \tilde{a}^{(n, \mu)}$ are respectively associated to $A_{N}(z ; n, \mu), \widetilde{A_{N}^{N}}(z ; n, \mu)$, for some fixed values of $N, \tilde{N}$, while the wavelet masks $b^{(n, \mu)}, \tilde{b}^{(n, \mu)}$ are the coefficients of the wavelet symbols obtained through (10).

Suppose $f$ is an input discrete signal represented by the coefficient sequence $c^{(m)}=\left(c_{j}^{(m)}: j \in \mathbb{Z}\right)$. Such a signal can be processed by the filterbank system through iterated low-pass/high-pass decomposition/reconstruction processes. In particular, the level-dependent decomposition scheme produces the 

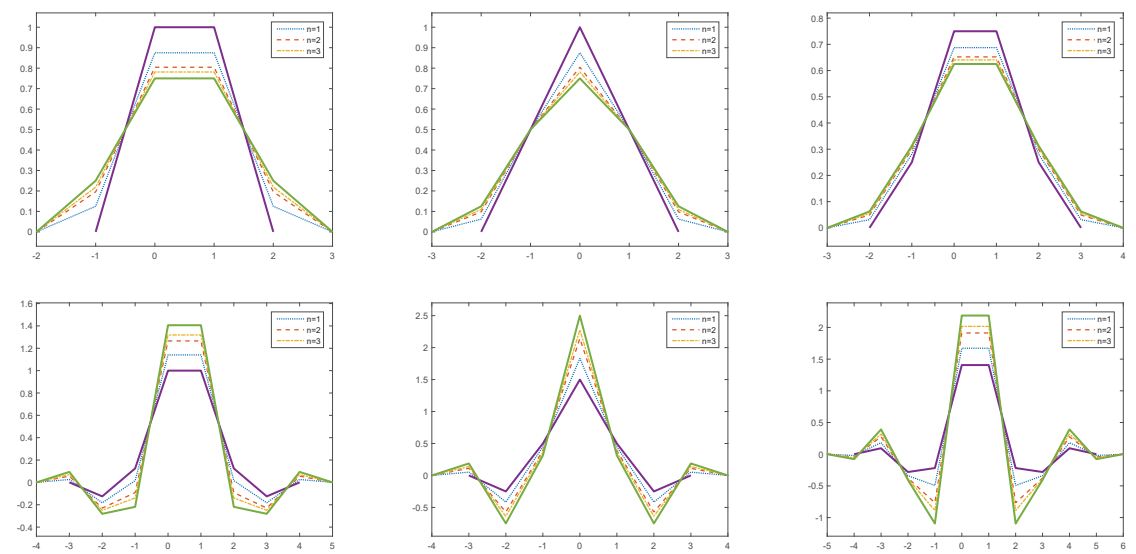

Figure 1: The primal filters (top) corresponding to $N=1, N=2, N=3$ and the respective associated duals (bottom) corresponding to $\tilde{N}=3, \tilde{N}=2, \tilde{N}=3$, for $n=1,2,3$. The parameter $\mu$ is fixed to 1.5. The two thick solid lines in each plot represent the limit B-spline filters.

sequences $\left(c^{(m-L)}, d^{(m-L)}, \ldots, d^{(m-1)}\right)$, up to a fixed level $L$, as

$$
\begin{aligned}
& c_{j}^{(n)}=\sum_{k \in \mathbb{Z}} \tilde{a}_{k-2 j}^{(n, \mu)} c_{k}^{(n+1)}, \\
& d_{j}^{(n)}=\sum_{k \in \mathbb{Z}} \tilde{b}_{k-2 j}^{(n, \mu)} c_{k}^{(n+1)},
\end{aligned}
$$

while the level-dependent reconstruction scheme allows for the reconstruction of the original sequence by means of:

$$
c_{k}^{(n+1)}=\sum_{j \in \mathbb{Z}}\left(a_{k-2 j}^{(n, \mu)} c_{j}^{(n)}+b_{k-2 j}^{(n, \mu)} d_{j}^{(n)}\right), \quad k \in \mathbb{Z}, n=m-L, \ldots, m-1 .
$$

At each step of the decomposition/reconstruction schemes, the filter taps change according to the level (and to the parameter $\mu$ ), thus providing a nonstationary, in the sense of level-dependent, way to analyze and synthesize a signal.

The extension to the bivariate case is straightforward if we limit ourselves to the separable case, where the $2 \mathrm{D}$ filters are just tensor products of the univariate filters, which result in processing the data according to the coordinate axis directions.

We show now how a proper choice of the parameter $\mu$ in the level-dependent filterbanks can lead to better results in image processing applications than stationary filterbanks. In particular, our numerical tests illustrate the compression effectiveness of the proposed filters, when compared to their limit versions, namely the B-spline filters.

The $2 \mathrm{D}$ discrete decimated wavelet transform using the proposed nonstationary filters has been applied to two common and popular $512 \times 512 \times 8$ bits test 

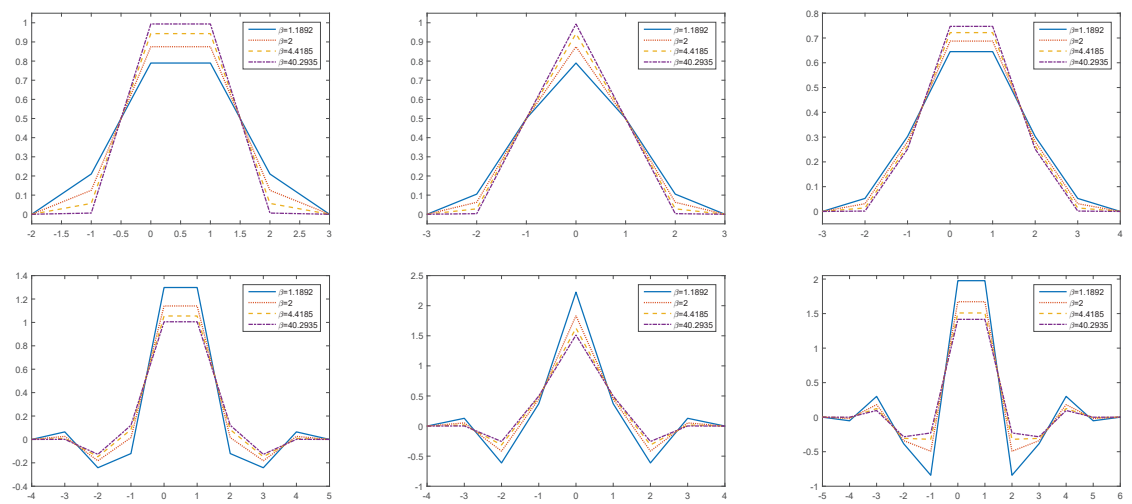

Figure 2: The primal filters (top) corresponding to $N=1, N=2, N=3$ and the respective associated duals (bottom) corresponding to $\tilde{N}=3, \tilde{N}=2, \tilde{N}=3$, for different values of the parameter $\beta$.

images: Lena and Barbara. They have been then approximated by retaining a small percentage (rate) of detail coefficients - the most significant coefficients have been preserved, the remaining ones have been set equal to zero. The same has been done using the limit B-splines filters. Results in Figure 3 refer to nonstationary filters with $N=1, \tilde{N}=3$ for different values of the parameter $\mu$ and starting level $L$; the rate has been fixed equal to $10 \%$. The approximation error has been measured in terms of Peak Signal-to-Noise Ratio (PSNR), i.e.

$$
\operatorname{PSNR}(I, \tilde{I})=10 \log _{10} \frac{255^{2}}{\operatorname{MSE}(I, \tilde{I})},
$$

where $I$ is the original image, $\tilde{I}$ is its approximation, and

$$
\operatorname{MSE}(I, \tilde{I})=\frac{1}{N_{x} N_{y}} \sum_{i=1}^{N_{x}} \sum_{j=1}^{N_{y}}(I(i, j)-\tilde{I}(i, j))^{2},
$$

denotes the Mean Squared Error between the two image matrices, of size $N_{x} \times$ $N_{y}$, evaluated at each pixel location $(i, j)$ - the higher the PSNR the better $\tilde{I}$ approximates $I$. As it can be observed, at a fixed rate (percentage of retained coefficients), the nonstationary filters (abbreviated as "NS-filters") show better reconstruction properties than the ones of limit B-splines filters for a wide range of $\mu$ values.

It is interesting to observe that for highly textured images, like Barbara image, also some negative values of the parameter $\mu$ preserve good reconstruction properties.

In order to further evaluate and test the compaction properties of the proposed NS-filters, they have been embedded in a well known compression algorithm, namely the Set Partitioning In Hierarchical Trees (SPIHT) [12], which 

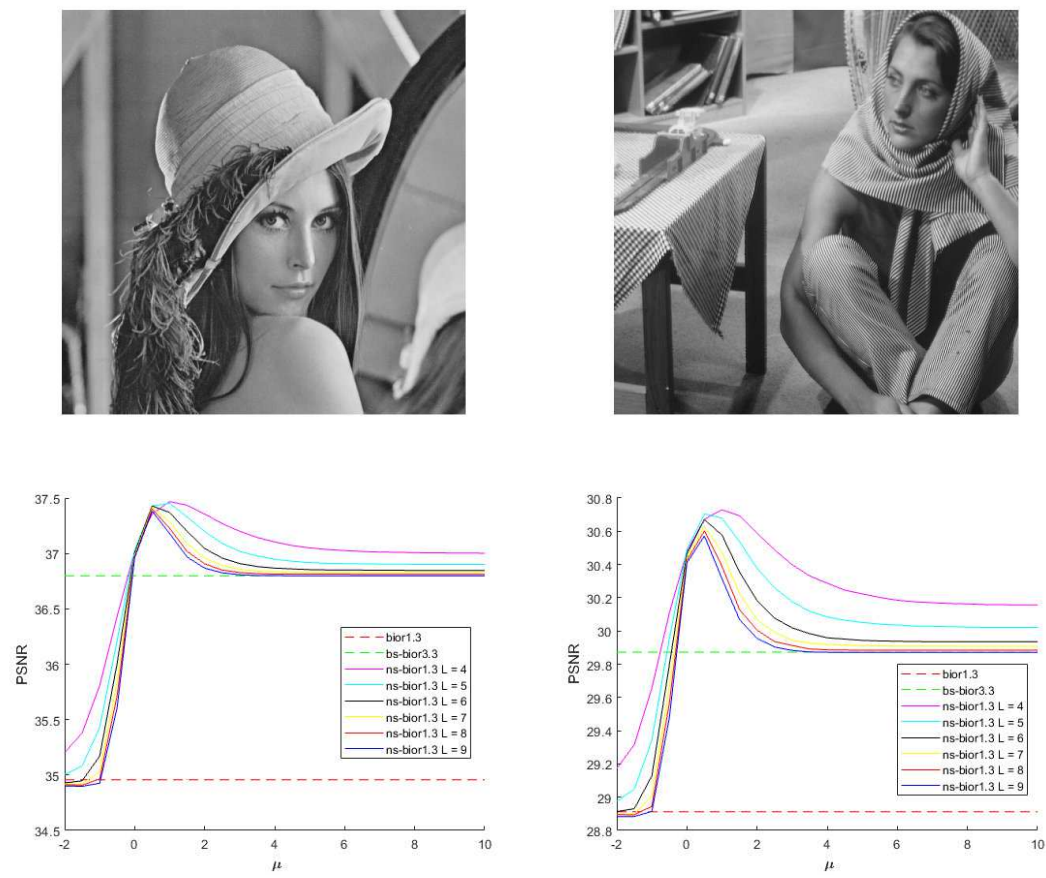

Figure 3: The original $512 \times 512 \times 8$ bits Lena (top left) and Barbara (top right) test images. The reconstruction error (bottom) (measured in terms of PSNR) obtained by retaining just the most significant $10 \%$ of detail coefficients of the multiresolution decomposition provided by nonstationary filters having $N=1, \tilde{N}=3$ and increasing $\mu$ value. Curve colors refer to a different starting level $L$ of the decomposition while dashed lines refer to the reconstruction error provided by the B-spline limit filters.

is a reference wavelet-based compression method. It mainly exploits both inter and intra scale relationships of wavelet coefficients, i.e. high amplitude coefficients are in correspondence to abrupt changes (main structures) in the original image and they show persistence properties along scale levels $[1,2]$.

Results have been measured in terms of PSNR and bits per pixel (bpp). The latter gives the compression rate and it is defined as

$$
b p p=\frac{8}{\mathrm{CF}},
$$

where $\mathrm{CF}$ is the compression factor, i.e. the ratio between the bits required to store the original uncompressed image and the ones required by its compressed version - the smaller the $b p p$ the higher the compression. For example, $b p p=0.5$ means that the compression factor is 16 , i.e. the compressed image is stored using $1 / 16$ of the bits required by the whole original image.

Figure 4 refers to $256 \times 256 \times 8$ bits Cameraman test image. As it can be observed, at the same compression rate $(b p p)$, NS-filters are able to reach 

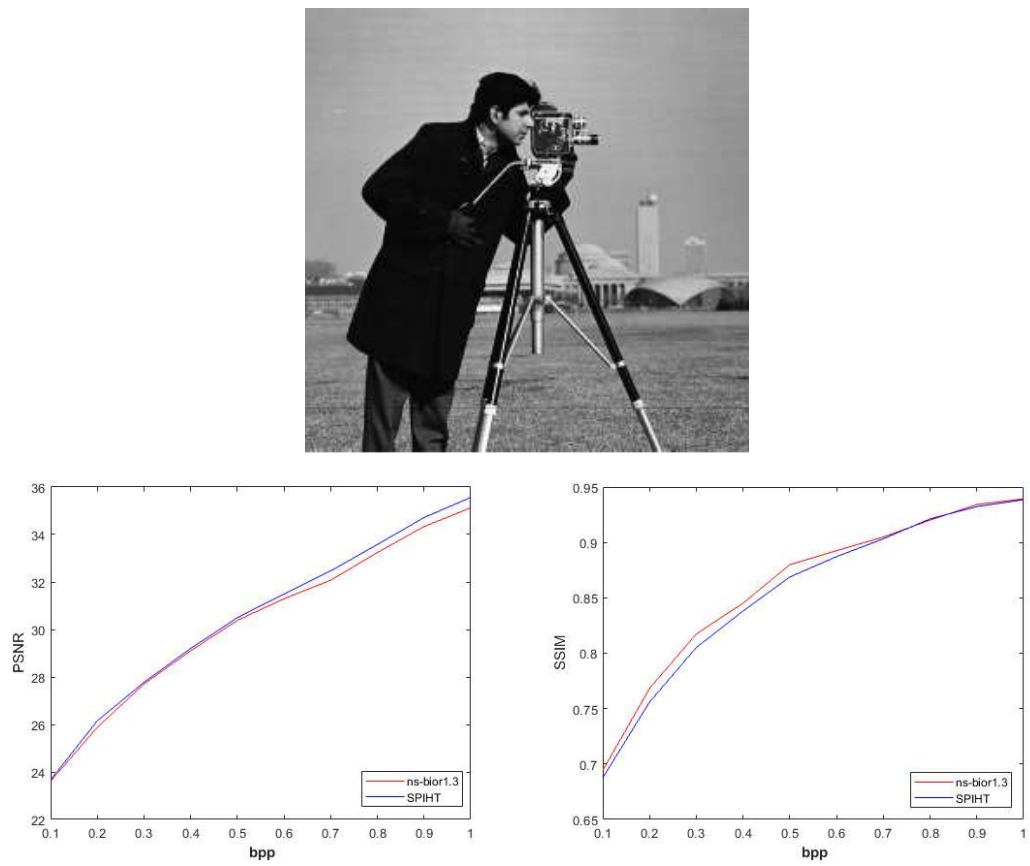

Figure 4: The original $256 \times 256 \times 8$ bits Cameraman test image $(t o p)$. PSNR versus bpp plot (bottom left) for compressed images with SPIHT algorithm using NS-filters having $N=1$, $\tilde{N}=3, \mu=0.1$ and $L=8$ (red line) and for compressed images with SPIHT algorithm using JP2K-like stationary biorthogonal filters (blue line). SSIM versus bpp plot (bottom right) for the same images.

PSNR values that are comparable to the one achieved by SPIHT using the JPEG2K biorthogonal stationary filters, even though the parameters of the algorithm have not been adapted to the nonstationary case. More interestingly, the visual quality of the decompressed images is better than the one provided by the standard SPIHT; hence, non stationarity correlates better with human perception, especially at low rates. In order to further stress this point, the Structural Similarity index (SSIM) [14] has been used for evaluating the quality of the decompressed images. SSIM is a full-reference image quality measure. It evaluates the visual difference between two images, $I$ and $\tilde{I}$, (or their subregions) as follows

$$
\operatorname{SSIM}(I, \tilde{I})=\frac{2 \mu_{I} \mu_{\tilde{I}}+C_{1}}{\mu_{I}^{2}+\mu_{\tilde{I}}^{2}+C_{1}} \frac{2 \sigma_{I} \sigma_{\tilde{I}}+C_{2}}{\sigma_{I}^{2}+\sigma_{\tilde{I}}^{2}+C_{2}} \frac{\sigma_{I \tilde{I}}+C_{3}}{\sigma_{I} \sigma_{\tilde{I}}+C_{3}},
$$

where $\mu_{*}$ and $\sigma_{*}$ respectively are the sample means and standard deviations of $*, \sigma_{I \tilde{I}}$ is the covariance between $I$ and $\tilde{I}$ while $C_{1}, C_{2}$ and $C_{3}$ are numerical stabilizing constants. The first two terms of SSIM measure the difference between the two images in terms of luminance mean and variance and they respectively 


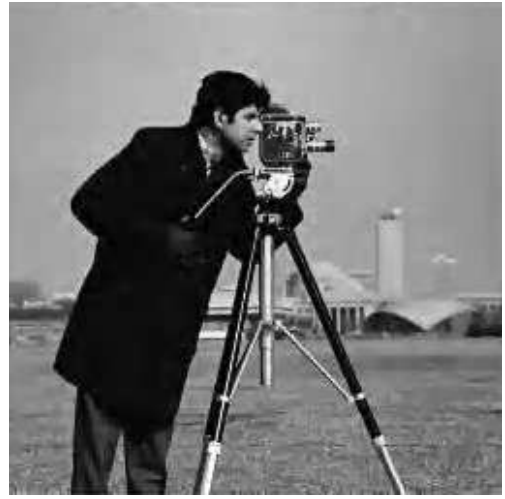

$\mathrm{PSNR}=30.38 ; \mathrm{SSIM}=0.8801$

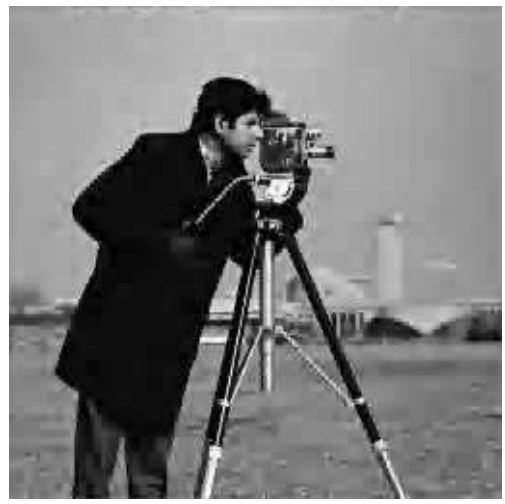

$\mathrm{PSNR}=27.70 ; \mathrm{SSIM}=0.8174$

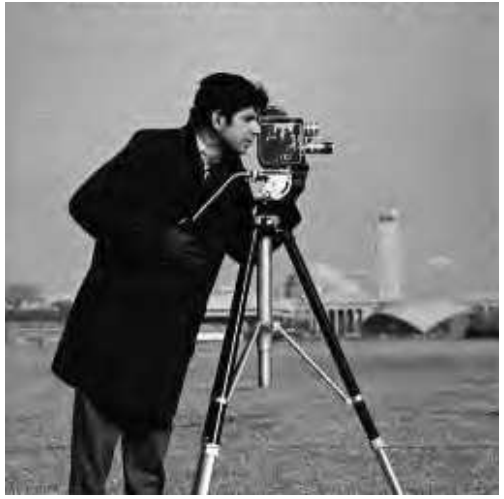

$\mathrm{PSNR}=30.49 ; \mathrm{SSIM}=0.8689$

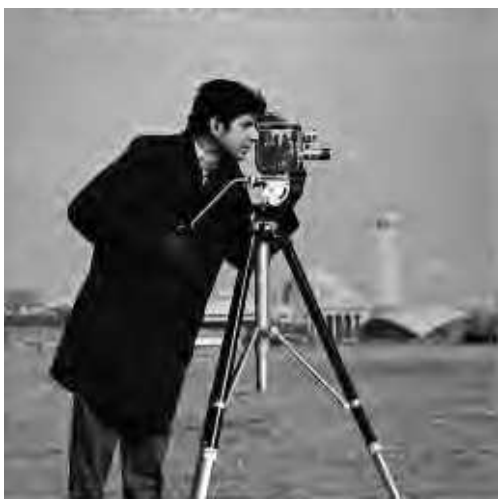

$\mathrm{PSNR}=27.77 ; \mathrm{SSIM}=0.8052$

Figure 5: Compression tests on the Cameraman image. Top) SPIHT compressed image at $b p p=0.5$ using: the nonstationary filters having $N=1, \tilde{N}=3, \mu=0.1$ and $L=8$ (left) and JP2K-like stationary biorthogonal filters (right). Bottom) SPIHT compressed image at $b p p=0.3$ using: the nonstationary filters having $N=1, \tilde{N}=3, \mu=0.1$ and $L=8$ (left) and JP2K-like stationary biorthogonal filters (right).

replicate two typical and basic mechanisms of human vision: luminance adaptation and contrast masking; the third term of SSIM measures the structural difference in terms of images covariance. SSIM outputs a number less than one: the closer to 1 this value, the better the image quality (i.e. $\tilde{I}$ visually resembles $I)$.

As it can be observed in Figure 4 (bottom right panel) NS-filters provide better SSIM values for the compressed images; hence, they are able to provide images with a better visual quality. In order to allow a visual inspection of the output images, in Figure 5 the ones corresponding to $b p p=0.5$ are given. The visual quality of the NS image is better than the one provided by SPIHT using standard stationary filters. For example, the highest building on the 

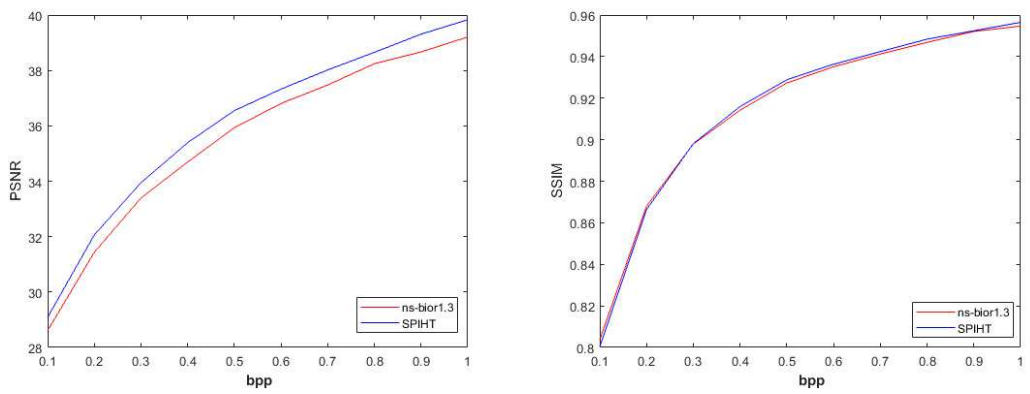

Figure 6: Test on the Lena image: PSNR versus bpp plot (left) for compressed images with SPIHT algorithm using nonstationary filters having $N=2, \tilde{N}=4, \mu=-0.5$ and $L=$ 6 (red line) and for compressed images with SPIHT algorithm using JP2K-like stationary biorthogonal filters (blue line). SSIM versus bpp plot (right) for the same images.

right of the scene is well reconstructed by NS-filters while it is blurred in the standard SPIHT image; in addition, some ringing effects around cameraman profile are considerably reduced in the nonstationary filters image while textures reconstruction is comparable. These observations are even more valid for lower rates, as shown in the same figure.

The better reconstruction of the discontinuities is the main advantage provided by non stationarity; the $\mu$ value regulates the convergence to smoother filters level by level and then it better allows us to isolate discontinuities as well as to emphasize persistence property of wavelet coefficients through scales. As a result, parent-child relationships, which represent the key issue of zero-tree based schemes, are better captured, preventing the effects of thresholding and quantization.

It is also worth outlining that, even when the difference in terms of PSNR between the nonstationary filters-based SPIHT image and the standard SPIHT one is higher, as in Figure 6, their visual quality is still comparable; in addition, the visual quality of NS-filters image results better at lower rates - this confirms the good correlation of nonstationary filters with the human visual system.

\section{Conclusion}

We have presented the construction of a family of biorthogonal wavelet filters generating level-dependent MRAs. Such filters have been derived from a class of convergent nonstationary subdivision schemes, corresponding, in the limit cases, to B-spline schemes. We have shown that the parametric form and the level-dependency of such filters allow for their better performance in comparison with B-spline filters in some image compression tests. The application of the proposed filters to other problems in image processing (for example denoising) is under investigation. 
A still open question is to find a strategy for automatically choosing the parameter $\mu$ according to the nature of the input data and to the application we are dealing with. This will be done in a forthcoming paper.

\section{Acknowledgment}

The authors would like to thank the anonymous referees for their helpful suggestions.

This work was partially supported by grants of University of Roma "La Sapienza", Ricerca Scientifica 2017, and by the 2018 INdAM-GNCS Project: "Sviluppo di modelli e metodi computazionali per l'elaborazione di segnali e immagini".

\section{References}

[1] V. Bruni, B. Piccoli, D. Vitulano, Time scale dependencies for image compression, J. Multimed., vol. 1, 44-55 (2006).

[2] V. Bruni, B. Piccoli, D. Vitulano, A fast computation method for time-scale signal denoising, Signal Image Video Process., vol. 3, 63-83 (2009).

[3] M. Cotronei, N. Sissouno, A note on Hermite multiwavelets with polynomial and exponential vanishing moments, Appl. Numer. Math., vol. 120, 21-34 (2017).

[4] A. Cohen, I. Daubechies, J.-C. Feauveau, Biorthogonal bases of compactly supported wavelets, Commun. Pure Appl. Math., vol. 45, 484-560 (1992).

[5] C. Conti, L. Gori, F. Pitolli, Totally positive functions through nonstationary subdivision schemes, J. Comput. Appl. Math., vol. 200, 255-265 (2007).

[6] M. Cotronei, M.L. Lo Cascio, A method for the construction of families of biorthogonal filters with prescribed properties, Adv. Comput. Math., vol. 17, 199-210 (2002).

[7] N. Dyn, D. Levin, Subdivision schemes in geometric modelling, Acta Numerica, 1-72, 2002.

[8] L. Gori, F. Pitolli, A class of totally positive refinable functions, Rend. Mat. Serie VII, vol. 20, 305-322 (2000).

[9] L. Gori, F. Pitolli, Nonstationary subdivision schemes and totally positive refinable functions, in Approximation Theory XII: San Antonio 2007, M. Neamtu and L.L. Schumaker (eds.), 169-180 (2008).

[10] L. Gori, F. Pitolli, Multiresolution analyses originated from nonstationary subdivision schemes, J. Comput. Appl. Math., vol. 221, 406-415 (2008). 
[11] F. Pitolli, Bell-shaped nonstationary refinable ripplets, Adv. Comput. Math., vol. 42, 1427-1451 (2016).

[12] A. Said, W.A. Pearlman, A new, fast, and efficient image codec based on set partitioning in hierarchical trees, IEEE Trans. on Circuits and Systems for Video Technology, vol. 6, 243-250 (1996).

[13] C. Vonesch, T. Blu, M. Unser, Generalized Daubechies wavelet families, IEEE Trans. on Signal Processing, vol. 55, 4415-4429 (2007).

[14] Z. Wang, A.C. Bovik, H.R. Sheikh, E.P. Simoncelli, Image quality assessment: From error visibility to structural similarity, IEEE Trans. on Image Processing, vol. 16, 600-612 (2004). 